\title{
¿Es la grasa abdominal subcutánea un predictor de la variabilidad cardiaca en hombres con bajo riesgo metabólico?
}

\author{
Is the abdominal subcutaneous fat a predictor of heart \\ rate variability in men with low metabolic risk?
}

Angélica Avendaño V', Edgar A. Rodríguez $\mathrm{M}^{1}$, Adriana Urbina ${ }^{2}$

\begin{abstract}
Forma de citar: Avendaño A, Rodríguez M, Urbina A. ¿Es la grasa abdominal subcutánea un predictor de la variabilidad cardíaca en hombres con bajo riesgo metabólico?. Rev Univ Ind Santander Salud. 2016; 48(3): $341-351$. DOI: http://dx.doi.org/10.18273/revsal.v48n3-2016008 (c) (1) Ð
\end{abstract}

\section{RESUMEN}

Introducción: Aunque el sobrepeso/obesidad, adiposidad aumentada e inactividad física se asocian a menor variabilidad cardíaca y desregulación autonómica, no se ha explorado en detalle la relación entre estos factores en sujetos de bajo riesgo metabólico. Objetivo: Analizar el tipo y fuerza de asociación entre la variabilidad cardíaca en reposo y la adiposidad en hombres jóvenes, delgados y físicamente activos. Metodología: Estudio descriptivo transversal. Se analizaron los componentes espectrales de la variabilidad cardíaca en 24 hombres de $18-30$ años físicamente activos, con peso y adiposidad normales. Se realizaron análisis de regresión lineal múltiple para explicar la variabilidad cardíaca en función de la relación cintura/cadera, pliegue abdominal y actividad física. Resultados: El volumen de actividad física semanal se relacionó positivamente con RMSSD y HF, y negativamente con LF y LF/HF. La relación cintura/cadera y el pliegue abdominal se relacionaron positivamente con HF y negativamente con LF y LF/HF. En todos los casos, los coeficientes de regresión $\beta$ tipificados estuvieron entre 0,395 y 0,505 ( $<<0,05)$. El índice de grasa visceral calculado a partir de bioimpedancia no mostró relación alguna. Conclusiones: Los hallazgos aquí reportados son indicativos de que en hombres jóvenes, delgados y físicamente activos, la actividad física y la grasa subcutánea se relacionan positivamente con el tono vagal y negativamente con el tono simpático, lo que lleva a postular que la acumulación del exceso calórico en forma de tejido adiposo subcutáneo (y no visceral) podría ser un mecanismo de protección contra el desarrollo de síndrome metabólico en estos sujetos.

Palabras clave: Frecuencia cardíaca, sistema nervioso parasimpático, distribución de la grasa corporal, grasa subcutánea abdominal, ejercicio, sistema nervioso autónomo.

1. Universidad Pedagógica y Tecnológica de Colombia UPTC. Tunja, Boyacá.

2. Universidad del Rosario. Bogotá DC. Colombia

Correspondencia: Adriana Urbina. Dirección: Carrera 24 \#63C-69 Quintas Mutis, Bogotá DC. Correo electrónico: adriana.urbina@urosario. edu.co. Teléfono: 2970200 Ext. 3319. 


\section{ABSTRACT}

Objective: To measure the type and strength of the potential association between heart rate variability at rest and some indicators of adiposity in young, lean, and physically active men. Introduction: Although overweight/obesity, increased adiposity and physical inactivity are associated with decreased heart rate variability and autonomic dysregulation, the relationship between these factors has not been explored in detail in subjects with low metabolic risk: normal weight, low adiposity and physically active. Methodology: Cross-sectional analytical study. The spectral components of heart rate variability were analyzed in 24 physically active men, 18-30 years old, with normal weight and adiposity. Multiple linear regression analysis was performed to explain heart rate variability as a function of waist to hip ratio, abdominal skinfod and physical activity. Results: The volume of weekly physical activity was positively related to RMSSD and HF, and negatively with LF and LF/HF. Both the waist to hip ratio and abdominal skinfold were positively related with $\mathrm{HF}$, and negatively with LF and LF/HF. In all cases, the B regression standardized coefficients were between 0.395 and $0.505(\mathrm{p}<0.05)$. The visceral fat rating calculated from bioimpedance showed no relation. Conclusions: The findings reported here are indicative that in young, lean and physically active men, physical activity and subcutaneous fat are positively related to vagal tone and negatively with sympathetic tone. This leads us to postulate that the accumulation of caloric excess in the form of subcutaneous adipose tissue (not visceral) might be a protective mechanism against the development of metabolic syndrome in these subjects.

Keywords: Heart rate control, parasympathetic nervous system, body fat distribution, subcutaneous abdominal fat, exercise, autonomic nervous system.

\section{INTRODUCCIÓN}

Las enfermedades relacionadas con el sobrepeso y la obesidad van en aumento. La relación entre enfermedades cardiovasculares y obesidad está ampliamente documentada y ha tratado de explicarse mediante varios mecanismos fisiopatológicos incluyendo resistencia a la insulina, hipertensión y dislipidemia. Sin embargo, también se ha sugerido que la desregulación en la función autonómica puede explicar la ocurrencia aumentada de enfermedad cardiovascular en personas con sobrepeso y obesidad ${ }^{1}$. La variabilidad de la frecuencia cardíaca permite medir el efecto de la función autonómica sobre el corazón. Por este motivo, varios estudios se han dirigido al análisis de la variabilidad cardíaca en personas con sobrepeso u obesidad. En general, una variabilidad cardíaca disminuida se asocia a incremento de morbilidad y mortalidad en enfermedades cardiovasculares ${ }^{2}$. Varios indicadores de variabilidad cardíaca están alterados en las personas con sobrepeso u obesidad incluyendo variabilidad reducida, aumento de tono simpático y disminución de tono parasimpático ${ }^{3}$. Estas alteraciones se relacionan con el índice de masa corporal ${ }^{4}$, relación cintura/cadera ${ }^{5}$, grasa corporal $^{6}$ y grasa visceral ${ }^{7}$, pero no la grasa subcutánea ${ }^{8}$.

Por otra parte, la inactividad física es un problema de salud pública en aumento en el ámbito mundial, contribuyendo al desarrollo de enfermedades cardiovasculares y al incremento del riesgo prematuro de muerte9. La realización de actividad física propicia beneficios en la salud, tales como prevención de la diabetes tipo 2, hipertensión, obesidad, cáncer y enfermedad coronaria ${ }^{10}$. Las personas que realizan actividad física moderada o vigorosa presentan mayor variabilidad cardíaca comparadas con las físicamente inactivas. Incluso, los hombres con sobrepeso pero que realizan actividad física vigorosa muestran una variabilidad cardíaca similar a la de los sujetos con peso normal. Lo anterior sugiere que una mayor variabilidad cardíaca, reflejo de mejor regulación autonómica es un posible mecanismo que explica cómo la actividad física resulta en reducción del riesgo de enfermedad coronaria ${ }^{11}$.

Aunque está bien establecido que el sobrepeso/ obesidad, la adiposidad aumentada y la inactividad física se asocian a disminución de la variabilidad cardíaca y cambios en los componentes espectrales que reflejan desregulación autonómica, no se ha explorado en detalle la relación entre estos factores en los sujetos de bajo riesgo metabólico: peso normal, baja adiposidad y físicamente activos. El objetivo de este estudio fue analizar el tipo y fuerza de asociación entre la variabilidad cardíaca en reposo y la adiposidad en hombres jóvenes, delgados y físicamente activos.

\section{METODOLOGÍA}

\section{Sujetos de estudio}

Participaron 24 hombres aparentemente sanos entre 18 y 30 años. Un comité de ética institucional aprobó el protocolo experimental, y todos los sujetos participantes 
¿Es la grasa abdominal subcutánea un predictor de la variabilidad cardíaca en hombres con bajo riesgo metabólico?

proporcionaron su consentimiento informado. Ninguno de los individuos estaba consumiendo medicamentos con efecto sobre el sistema nervioso autónomo. Los sujetos se habían abstenido de consumir bebidas con cafeína y alcohol en las doce horas previas, y de realizar actividad física en las últimas 24 horas. Los sujetos fueron indagados respecto al sueño durante la noche anterior con el cuestionario "Karolinska Sleep Diary" (KSD). Los participantes acudieron el día del estudio en condiciones de ayuno, se les proporcionó una comida estándar y una hora después se realizaron las mediciones, todas entre 6:00 y 9:00 AM. Teniendo en cuenta que la actividad física modifica la composición corporal y la variabilidad cardíaca, se utilizó como criterio de inclusión realizar un volumen de actividad física de al menos 150 minutos/semana. Fueron considerados criterios de exclusión: tabaquismo, haber respondido positivamente alguno de los ítems del cuestionario de alistamiento para la actividad física (PAR-Q), padecer de trastornos cardiovasculares, pulmonares, metabólicos o disautonomía.

\section{Composición corporal}

Se midieron estatura y peso, y se calculó el índice de masa corporal (IMC). Se midieron los perímetros de cintura, abdomen y cadera con una cinta métrica (Myotape $^{\circledR}$, AccuFitness, USA; precisión un mm) y el pliegue abdominal con un adipómetro (Harpenden ${ }^{\circledR}$, Baty International, West Sussex, Reino Unido) en tres oportunidades y se registró el valor promedio; los coeficientes de variación de estas mediciones fueron inferiores a 1\%. Mediante impedancia bioeléctrica se midió el porcentaje de grasa corporal y se calculó el índice de grasa visceral mediante el algoritmo desarrollado por el fabricante (Tanita $^{\circledR}$ BC554 IRONMAN ${ }^{\circledR}$ GLASS, Tanita, Japón).

Variabilidad de la frecuencia cardíaca en posición supina y ortostatismo

Después de cinco minutos de reposo en posición supina (SUP), se midió la variabilidad de la frecuencia cardíaca durante cinco minutos y luego en posición de bipedestación durante cinco minutos más, mediante un monitor cardíaco (Polar ${ }^{\circledR}$ RS800CX, Polar Electro, Finlandia). Los registros de intervalos RR fueron descargados y se realizó corrección de errores con filtro moderado, mediante el software Polar Performance ${ }^{\circledR}$ versión 4.01.029. Con el fin de analizar la reproducibilidad dentro de cada registro, se determinaron los indicadores de variabilidad cardíaca en el dominio de tiempo y espectral en segmentos de 60,90 y 120 segundos consecutivos y se realizó análisis de correlación de Spearman, los coeficientes de determinación $\left(\mathrm{R}^{2}\right)$ estuvieron entre 0,70 y 0,94 . Los resultados presentados corresponden a los 180 segundos centrales del registro en cada posición. Se realizó el análisis en dominio de tiempo y espectral (método autoregresivo) con el software Kubios HRV versión 2.0 de la Universidad de Kuopio, Finlandia.

\section{Análisis estadístico}

La distribución de los datos se determinó mediante prueba de Shapiro-Wilk y los resultados se presentan como mediana \pm amplitud intercuartil. Las comparaciones entre posición supina y ortostatismo se realizaron mediante prueba de Wilcoxon para muestras relacionadas. Los análisis bivariados se realizaron con coeficientes de correlación de Spearman.

Las variables actividad física, LF/HF y RMSSD presentaron una distribución sesgada hacia la derecha, por lo cual se realizó transformación logarítmica para producir distribuciones normales. Se realizaron análisis de regresión lineal múltiple, seleccionando como variable dependiente las relacionadas con variabilidad cardíaca y como variables independientes la relación cintura/cadera, logaritmo del volumen de actividad física semanal y edad. Para la entrada/salida de variables se utilizó el método por pasos aplicando como criterio de entrada una probabilidad de $\mathrm{F}$ equivalente a $\mathrm{p} \leq 0,05$ y como criterio de salida $\mathrm{p} \geq 0,10$. Se evaluó la independencia de residuos mediante el estadístico de Durbin Watson cercano a 2,0. Se evaluó la homocedasticidad y linealidad de los residuos a partir de la gráfica de los residuos predichos vs observados, su distribución mediante la prueba de Shapiro-Wilk y se calculó el error cuadrático medio de los residuos. Se utilizó el paquete estadístico IBM SPSS versión 23.0 (Licencia de la Universidad del Rosario). Se estableció el nivel de significancia en $p<0,05$. La potencia de una muestra de 24 sujetos estudiados para detectar coeficientes de correlación superiores a 0,5 es de 73 a $82 \%$, de acuerdo con los cálculos realizados con el paquete Epidat versión 4.1.

\section{RESULTADOS}

\section{Indicadores de adiposidad de los participantes}

Las características generales de la muestra se resumen en la Tabla 1. Participaron en el estudio 24 hombres adultos jóvenes con edades entre 18 y 30 años físicamente activos (al menos 150 minutos/semana) ${ }^{12}$. 21 de 24 sujetos tuvieron IMC en el rango saludable 
$\left(18,5\right.$ a $\left.24,9 \mathrm{~kg} / \mathrm{m}^{2}\right)$ con porcentajes de grasa entre 6 y $19 \%$. Mientras que tres de 24 tuvieron el IMC en el rango entre 25 y $25,9 \mathrm{~kg} / \mathrm{m}^{2}$, pero con porcentajes de grasa entre 9 y $22 \%$, motivo por el cual no pueden ser clasificados como individuos con sobrepeso o adiposidad aumentada ${ }^{13}$. Otros indicadores que permiten valorar indirectamente adiposidad abdominal permiten descartar la presencia de obesidad abdominal en la muestra de estudio: el índice de grasa visceral estuvo entre uno y cuatro (valores normales entre 1-12) $)^{14}$; el perímetro abdominal estuvo entre 73 y 87 $\mathrm{cm}$; y la relación cintura/cadera entre 0,74 y $0,94^{15}$. Por lo tanto, todos los sujetos de la muestra pueden ser considerados como adultos jóvenes, físicamente activos y sin obesidad abdominal. Por otra parte, teniendo en cuenta que la cantidad y calidad de sueño puede afectar las mediciones de variabilidad de la frecuencia cardiaca, se utilizó el cuestionario Karolinska Sleep Diary, cuyos resultados mostraron tiempos de sueño entre cuatro y ocho horas. Ya que la latencia de sueño estuvo entre dos y 60 minutos, se pudo descartar la ocurrencia de insomnio de conciliación que pudiera afectar las mediciones de variabilidad cardíaca ${ }^{16}$.

TABLA 1. Características generales de la muestra $(n=24)$.

\begin{tabular}{lcc}
\hline & Mediana & $\begin{array}{c}\text { Amplitud } \\
\text { intercuartil }\end{array}$ \\
\hline Edad & 22 & 7 \\
Actividad física (min/semana) & 260 & 640 \\
Talla (m) & 1,72 & 0,06 \\
Peso (kg) & 68,5 & 11,7 \\
Índice de masa corporal (kg/m²) & 22,1 & 3,3 \\
Grasa (\%) & 14,0 & 6,8 \\
Índice de grasa visceral & 2 & 2 \\
Pliegue abdominal (mm) & 12,4 & 9,9 \\
Perímetro abdominal (cm) & 78,5 & 6,5 \\
Relación cintura/cadera & 0,81 & 0,04 \\
Tiempo de sueño (min) & 390 & 80 \\
Latencia de sueño (min) & 20 & 20 \\
\hline
\end{tabular}

\section{Variabilidad cardíaca en posición supina y} ortostatismo

El análisis de la variabilidad cardíaca en reposo mostró un comportamiento característico en posición supina y ortostatismo. Con el cambio de posición se observó aumento significativo de la frecuencia cardíaca con disminución de SDNN, RMSSD, así como de intervalos RR sucesivos que difieren en más de $50 \mathrm{~ms}$ (NN50) y el porcentaje de intervalos RR sucesivos con diferencia mayor a $50 \mathrm{~ms}$ (pNN50). En cuanto a los componentes espectrales, se observó aumento de HF con disminución de LF y LF/HF. Estos hallazgos son consistentes con activación simpática e inhibición vagal en ortostatismo con respecto a la posición supina (Tabla 2) ${ }^{17}$.

TABLA 2. Variabilidad cardíaca en reposo en posición supina y ortostatismo.

\begin{tabular}{lcccc}
\hline \multirow{2}{*}{ Variable } & \multicolumn{2}{c}{ Decúbito } & \multicolumn{2}{c}{ Ortostatismo } \\
\cline { 2 - 5 } & Mediana $\begin{array}{c}\text { Amplitud } \\
\text { intercuartil }\end{array}$ & Mediana & $\begin{array}{c}\text { Amplitud } \\
\text { intercuartil }\end{array}$ \\
\hline $\begin{array}{l}\text { Frecuencia } \\
\text { cardíaca } \\
\text { (min-1) }\end{array}$ & 64,0 & 16,7 & $81,0 *$ & 15,1 \\
RR (s) & 949,9 & 252,4 & $744,0 *$ & 132,4 \\
SDNN & 81,2 & 53,4 & $53,2 *$ & 25,0 \\
RMSSD & 80,3 & 43,0 & $24,6 *$ & 14,7 \\
NN50 & 88 & 59 & $11,0 *$ & 26,0 \\
pNN50 (\%) & 53,0 & 35,9 & $5,0 *$ & 10,9 \\
LF (nu) & 51,2 & 20,9 & $81,3 *$ & 24,7 \\
HF (nu) & 48,8 & 20,9 & $18,7 *$ & 24,7 \\
LF/HF (\%) & 1,0 & 0,9 & $4,3 *$ & 4,3 \\
\hline RR: intervalos & & & & \\
\end{tabular}

RR: intervalos RR; SDNN: desviación estándar de todos los intervalos RR; RMSSD: raíz cuadrada de la diferencias entre intervalos RR sucesivos elevadas al cuadrado; NN50: número de intervalos RR sucesivos que difieren entre sí por más de $50 \mathrm{~ms}$; pNN50: porcentaje de intervalos RR sucesivos que difieren entre sí por más de $50 \mathrm{~ms}$; LF: componente de baja frecuencia; HF: componente de alta frecuencia; nu: unidades normalizadas.

*Diferencias estadísticamente significativas $(\mathrm{p}<0,01)$.

\section{Relación entre variabilidad cardíaca e indicadores de adiposidad}

No se encontró correlación de la variabilidad cardíaca con el índice de masa corporal, porcentaje de grasa corporal, grasa visceral ni perímetro abdominal $(\mathrm{p}>0,05)$, pero sí con la relación cintura/ cadera, actividad física y edad. Por este motivo se realizaron análisis de regresión lineal múltiple con el fin de encontrar modelos explicativos para diversos indicadores de variabilidad cardíaca como variable dependiente. En todos los modelos multivariados, la variable edad fue excluida toda vez que la probabilidad $F$ fue equivalente a $\mathrm{p} \geq 0,10$. Se observó la existencia de una relación entre las variables que viene explicada por la ecuación:

$$
y=4,928-3,961 X_{1}-0,434 X_{2}
$$

Donde $y$ es el logaritmo de la relación LF/HF en ortostatismo, $X_{1}$ es la relación cintura/cadera y $X_{2}$ es el logaritmo del volumen de actividad física (minutos/ semana). 
¿Es la grasa abdominal subcutánea un predictor de la variabilidad cardíaca en hombres con bajo riesgo metabólico?

El coeficiente de determinación fue 0,415 y el error cuadrático medio de 0,0749. En la Tabla 3 se muestran los coeficientes tipificados y sus valores de probabilidad.

TABLA 3. Coeficientes B para Log LF/HF en ortostatismo como variable dependiente.

\begin{tabular}{lcccc}
\hline & $\mathbf{B}$ & $\mathbf{S E}^{\mathbf{a}} \mathbf{B}$ & $\begin{array}{c}\mathbf{B} \\
\text { tipificado }\end{array}$ & $\boldsymbol{P}$ \\
\hline (Constante) & 4,928 & 1,245 & & 0,001 \\
Relación cintura/cadera & $-3,961$ & 1,445 & $-0,471$ & 0,013 \\
$\log ($ actividad física) & $-0,434$ & 0,185 & $-0,402$ & 0,030 \\
\hline
\end{tabular}

${ }^{a}$ Error estándar.

Teniendo en cuenta que los coeficientes B tipificados permiten comparar variables con diferentes escalas, es posible concluir que la variable Log LF/HF en ortostatismo presenta una mayor contribución explicada por la relación cintura/cadera, con respecto a actividad física (coeficientes tipificados $-0,471 \mathrm{y}$ $-0,402$, respectivamente).

En la Tabla 4 se presenta un resumen de los indicadores de variabilidad cardíaca en reposo que pueden ser explicados por la relación cintura/cadera, la actividad física o por ambas variables. Para poder evaluar la contribución relativa de cada una de estas variables predictoras sobre el comportamiento de la variabilidad cardíaca, los coeficientes de regresión se expresan como coeficientes tipificados.

TABLA 4. Coeficientes tipificados $B$ en los análisis de regresión lineal que permiten explicar el comportamiento de la variabilidad cardíaca (variables dependientes) en función de la relación cintura/cadera y la actividad física (variables predictoras).

\begin{tabular}{lcc}
\hline & $\begin{array}{c}\text { Relación } \\
\text { cintura/cadera }\end{array}$ & $\begin{array}{c}\text { Log } \\
\text { (actividad } \\
\text { física) }\end{array}$ \\
\hline LF decúbito & $-0,480 *$ & - \\
LF ortostatismo & $-0,484 *$ & $-0,394^{*}$ \\
HF decúbito & $0,480 *$ & - \\
HF ortostatismo & $0,484 *$ & $0,395 *$ \\
Log LF/HF decúbito & $-0,452 *$ & - \\
Log LF/HF ortostatismo & $-0,471 *$ & $-0,402 *$ \\
Log RMSSD decúbito & - & $0,409 *$ \\
Log RMSSD ortostatismo & - & $0,505 *$ \\
\hline
\end{tabular}

*Indica que el valor de los coeficientes es significativamente diferente de $0(\mathrm{p}<0,05)$.

Se excluyeron las variables con una probabilidad de $\mathrm{F}$ equivalente a $\mathrm{p} \geq 0,10$; en todos los casos la variable edad resultó excluida con base en este criterio.
Teniendo en cuenta que la relación cintura/cadera es un indicador de adiposidad abdominal tanto subcutánea como intra-abdominal, y con el fin de determinar la contribución de la grasa subcutánea, se realizaron también análisis de regresión múltiple incorporando la variable pliegue abdominal en reemplazo de la relación cintura/cadera dentro de variables predictoras, encontrando hallazgos similares: relación positiva con HF y negativa con LF y LF/HF sin modificación significativa de los coeficientes para actividad física (Tabla 5). Posteriormente, con el fin de analizar la contribución de la adiposidad intra-abdominal, se realizó el mismo procedimiento reemplazando la relación cintura/cadera por la variable índice de grasa visceral en todos los modelos multivariados, pero resultó excluida en todos los casos. Lo anterior sugiere que el papel de la relación cintura/cadera como variable explicativa de la variabilidad cardíaca viene dado principalmente por la acumulación de tejido adiposo subcutáneo más que intra-abdominal.

TABLA 5. Coeficientes tipificados $B$ en los análisis de regresión lineal que permiten explicar el comportamiento de la variabilidad cardíaca (variables dependientes) en función del pliegue abdominal y la actividad física (variables predictoras).

\begin{tabular}{lcc}
\hline & $\begin{array}{c}\text { Pliegue } \\
\text { abdominal }\end{array}$ & $\begin{array}{c}\text { Log } \\
\text { (actividad física) }\end{array}$ \\
\hline LF decúbito & $-0,438 *$ & - \\
LF ortostatismo & - & $-0,436^{*}$ \\
HF decúbito & $0,492 *$ & - \\
HF ortostatismo & $0,484^{*}$ & $0,395 *$ \\
Log LF/HF decúbito & $-0,414^{*}$ & - \\
Log LF/HF ortostatismo & - & $-0,442 *$ \\
Log RMSSD decúbito & - & $0,409 *$ \\
Log RMSSD ortostatismo & - & $0,505 *$ \\
\hline
\end{tabular}

*Indica que el valor de los coeficientes es significativamente diferente de $0(\mathrm{p}<0,05)$.

Se excluyeron las variables con una probabilidad de $\mathrm{F}$ equivalente a $\mathrm{p} \geq 0,10$; en todos los casos la variable edad resultó excluida con base en este criterio.

\section{DISCUSIÓN}

Los sujetos con sobrepeso/obesidad y adiposidad aumentada tienen menor variabilidad cardíaca en reposo, mayor tono simpático y menor tono vagal ${ }^{18-20}$. De manera similar, los sujetos físicamente inactivos con peso normal muestran menor variabilidad cardíaca en reposo con predominio de tono simpático y tono vagal disminuido ${ }^{19,20}$. Es decir, tanto el sobrepeso/obesidad como la inactividad física tienen efectos similares y 
deletéreos sobre la variabilidad cardíaca, los cuales podrían ser sinérgicos ${ }^{20}$. Teniendo en cuenta que la realización de actividad física (al menos 150 minutos/ semana) se recomienda para inducir beneficios en la salud y proteger contra el desarrollo de enfermedades crónicas no transmisibles ${ }^{12}$, es deseable estudiar la relación entre adiposidad y variabilidad cardíaca en sujetos físicamente activos. Para explorar el tipo y fuerza de tal asociación, el presente estudio utilizó una muestra de 24 hombres entre 18 y 30 años físicamente activos y sin obesidad abdominal.

Si bien los parámetros de variabilidad cardíaca en reposo en posición supina han mostrado ser útiles para evaluar la función del sistema nervioso autónomo y sirven como predictores de morbilidad y mortalidad ${ }^{21}$, los registros en esta posición se caracterizan por "saturación parasimpática" haciendo difícil encontrar diferencias significativas ${ }^{22}$. Por lo anterior, en este estudio se analizó la variabilidad cardíaca tanto en posición supina como ortostatismo. En estas condiciones de medición, nuestros sujetos de estudio mostraron el comportamiento típico en respuesta al estímulo ortostático consistente en activación simpática e inhibición vagal ${ }^{17}$.

Para abordar la cuestión de la relación existente entre variabilidad cardíaca y adiposidad, es necesario tener en cuenta confusores tales como sexo, edad y actividad física. El efecto de estas 3 variables se controló en el diseño mismo del estudio puesto que sólo se incluyeron hombres entre 18 y 30 años que realizaran al menos 150 minuto/semana de actividad física moderada/ vigorosa. A pesar de que en los análisis iniciales de correlación bivariada (Spearman) algunos parámetros de variabilidad cardíaca se correlacionaron con la edad, cuando esta variable fue incorporada para ajuste en los análisis de regresión múltiple resultó excluida. Lo anterior, es indicativo de que en el grupo de edad evaluado (18-30 años) los cambios en variabilidad asociados con la edad no son significativos como sí lo son en la edad mediana y avanzada ${ }^{23,24}$.

Se observó relación entre volumen de actividad física y varios indicadores de variabilidad cardíaca. Se encontró relación directa con RMSSD en posición supina, lo sugiere que el incremento del volumen de actividad física se relaciona con variabilidad cardíaca aumentada e incremento del tono vagal en reposo. De la misma manera, en ortostatismo se relacionó positivamente con HF y RMSSD, y negativamente con LF y FL/HF, lo que indica que los sujetos más activos físicamente presentan también en posición de bipedestación un mayor tono vagal acompañado de menor tono simpático. Estos hallazgos son similares a los encontrados por otros estudios $^{24,25}$ y podrían ser uno de los mecanismos protectores de la actividad física sobre morbilidad y mortalidad cardíaca ${ }^{21}$.

Al analizar la relación entre variabilidad cardíaca y adiposidad no se encontró correlación con parámetros como IMC, porcentaje de grasa, índice de grasa visceral ni perímetro abdominal, pero sí con la relación cintura/ cadera. Varios estudios han mostrado que el IMC puede tener baja sensibilidad para la detección de bajo peso y sobrepeso/obesidad, especialmente en hombres, en personas con buen desarrollo muscular y en edad avanzada $^{26-29}$. El porcentaje de grasa y perímetro abdominal son indicadores de adiposidad total, que sin embargo, están influenciados por la adiposidad abdominal ${ }^{30}$. Por su parte, la relación cintura/cadera es un indicador tanto de tejido adiposo subcutáneo como intra-abdominal ${ }^{15,31}$ y debido a que puede medirse con mayor exactitud que los pliegues cutáneos se utiliza ampliamente en estudios poblacionales ${ }^{15}$. Para fines diagnósticos y de prevención primaria y secundaria, la relación cintura/cadera ha mostrado buena sensibilidad y especificidad, las cuales son dependientes del punto de corte empleado según sexo y etnia ${ }^{32}$. En el presente estudio, la relación cintura/cadera al igual que el pliegue abdominal mostraron ser predictores del comportamiento de los componentes espectrales de la variabilidad cardíaca tanto en posición supina como en ortostatismo. Se observó relación positiva con HF y negativa con LF y LF/HF, lo que significa que en nuestra muestra de hombres jóvenes físicamente activos y sin sobrepeso, la adiposidad central subcutánea se relaciona negativamente con el tono simpático y positivamente con el tono vagal. Estos hallazgos son similares a los informados en adolescentes blancos ${ }^{33,34}$ y en hombres de peso normal pero de edad mediana y avanzada ${ }^{36,24}$.

Teniendo en cuenta que la relación cintura/cadera como indicador de adiposidad abdominal está influenciada por los depósitos de tejido adiposo tanto a nivel subcutáneo como intra-abdominal, se quiso explorar la potencial asociación entre la variabilidad cardíaca y el pliegue abdominal como indicador de grasa subcutánea y con el índice de grasa visceral proporcionado por el equipo de bioimpedancia (Tanita ${ }^{\circledR}$ BC554 IRONMAN ${ }^{\circledR}$ GLASS, Tanita, Japón). Con esta aproximación, se encontró que el pliegue abdominal se comporta como predictor de la variabilidad cardiaca de la misma manera que lo hace la relación cintura/cadera y sin modificar el efecto de la actividad física, mientras que el índice de grasa visceral no lo hace. Este hallazgo lleva a postular que en este 
¿Es la grasa abdominal subcutánea un predictor de la variabilidad cardíaca en hombres con bajo riesgo metabólico?

estudio, el marcador de adiposidad que es predictor de variabilidad cardíaca es la grasa subcutánea, la cual, se relaciona directamente con el componente vagal e inversamente con el simpático, comportamiento que se considera de protección cardiovascular ${ }^{21,37}$.

La inervación simpática y parasimpática del tejido adiposo ha sido estudiada en modelos animales en ratones y se ha documentado que mientras que la inervación simpática aumenta el número de adipocitos ${ }^{38}$, la parasimpática incrementa la sensibilidad a la insulina y la expresión de leptina y resistina ${ }^{39}$. Por otro lado, ya en humanos, ensayos en pacientes obesos que son sometidos a gastrectomía, aquellos a los que también se les realiza vagotomía muestran reducción significativa del volumen de grasa visceral seis meses después de la cirugía comparados con pacientes con gastrectomía sin vagotomía, sugiriendo que la inervación vagal desempeña un papel para evitar el depósito de grasa visceral en personas obesas ${ }^{40}$.

Adicionalmente, el denominado modelo del exceso de lípidos y grasa ectópica trata de explicar las interacciones entre grasa subcutánea y visceral en la patogénesis del síndrome metabólico. De acuerdo con este modelo, aunque la mayor parte de la energía corporal es almacenada en forma de grasa subcutánea, algunos individuos pueden almacenar cantidades sustanciales de tejido adiposo en su cavidad abdominal y ésta se correlaciona de manera significativa con las anormalidades cardio-metabólicas, de manera independiente de la grasa corporal total y grasa subcutánea ${ }^{41-43}$. El exceso de grasa visceral puede ser un marcador de disfuncionalidad del tejido adiposo, el cual no es capaz de almacenar adecuadamente el exceso calórico y que determina la susceptibilidad para desarrollar síndrome metabólico. Varios estudios sugieren que cuando el exceso calórico puede ser depositado en forma de tejido adiposo subcutáneo sensible a la insulina (capaz de expandirse mediante hiperplasia), el individuo estará protegido contra el desarrollo de síndrome metabólico ${ }^{44-47}$. No obstante, en casos en los cuales el tejido adiposo está ausente, deficiente o es resistente a la insulina y tiene una capacidad limitada para almacenar el exceso de energía (tejido adiposo hipertrófico), el exceso de triglicéridos será depositado en lugares indeseables como hígado, corazón, músculo esquelético y en el tejido adiposo visceral, fenómeno conocido como depósito de grasa ectópica ${ }^{48,49}$. Algunos factores que se asocian con acumulación preferencial de grasa a nivel visceral y resistencia a la insulina incluyen, pero no se limitan a tabaquismo, susceptibilidad genética documentada a la obesidad abdominal y un perfil neuroendocrino permisivo relacionado con respuesta maladaptativa al estrés (hiperactivación del eje hipotalámo-hipofisiarioadrenal). Las consecuencias metabólicas de este defecto de depósito de exceso calórico resultan en obesidad abdominal, resistencia a la insulina, dislipidemia aterogénica y un estado inflamatorio protrombótico, todos los cuales definen el síndrome metabólico ${ }^{50}$. En concordancia con el anterior modelo, algunos estudios han mostrado que dentro del tejido adiposo subcutáneo existen dos compartimentos diferenciados y separados por la fascia superficialis, por un lado el tejido subcutáneo profundo, con predominio proinflamatorio y determinante del síndrome metabólico, que se comporta de manera similar al tejido adiposo visceral y el tejido subcutáneo superficial que es el que potencialmente tendría el efecto protector que se ha discutido anteriormente ${ }^{51,52}$.

En la anterior línea de razonamiento, puede postularse que en nuestra muestra de estudio el hecho de que el pliegue abdominal como indicador de grasa subcutánea, se relacione positivamente con el tono vagal y negativamente con el tono simpático sugiere que en hombres jóvenes, delgados y físicamente activos, la posibilidad de acumular el exceso calórico en forma de tejido adiposo subcutáneo (sensible a la insulina) puede ser un mecanismo de protección contra el depósito de grasa intra-abdominal, el desarrollo de síndrome metabólico y se asocia a un adecuado balance autonómico ${ }^{47}$ (Figura 1). Por el contrario, con el aumento de la edad, un estilo de vida sedentario o el abandono de un estilo físicamente activo y la ganancia excesiva de peso, se desarrollaría resistencia a la insulina, que entre muchas otras, conduce a disfuncionalidad del tejido adiposo, el cual con una capacidad limitada para depositar el exceso calórico a nivel subcutáneo, empieza a depositarse de manera ectópica a nivel visceral contribuyendo al desarrollo de síndrome metabólico (independientemente de la grasa corporal total y subcutánea $)^{41-43}$ y se asocia a desregulación autonómica con predominio simpático ${ }^{53}$. Esta situación desfavorable podría ser intervenida a través de factores modificables, como por ejemplo la realización de actividad física, restricción calórica, disminución de peso, e inclusive con la modulación farmacológica de la sensibilidad a la insulina (Figura 1) ${ }^{54}$.

Las principales limitaciones de este estudio incluyen el diseño de tipo observacional transversal, el tamaño de muestra reducido y no haber controlado la intensidad de actividad física. Se sugiere conducir nuevos estudios con 
tamaños muestrales mayores; emplear cuestionarios de actividad física como por ejemplo IPAQ que permitan controlar no sólo el volumen sino también la intensidad; evaluar la adiposidad abdominal subcutánea y visceral con métodos más robustos, por ejemplo tomografía axial computarizada o resonancia nuclear magnética.

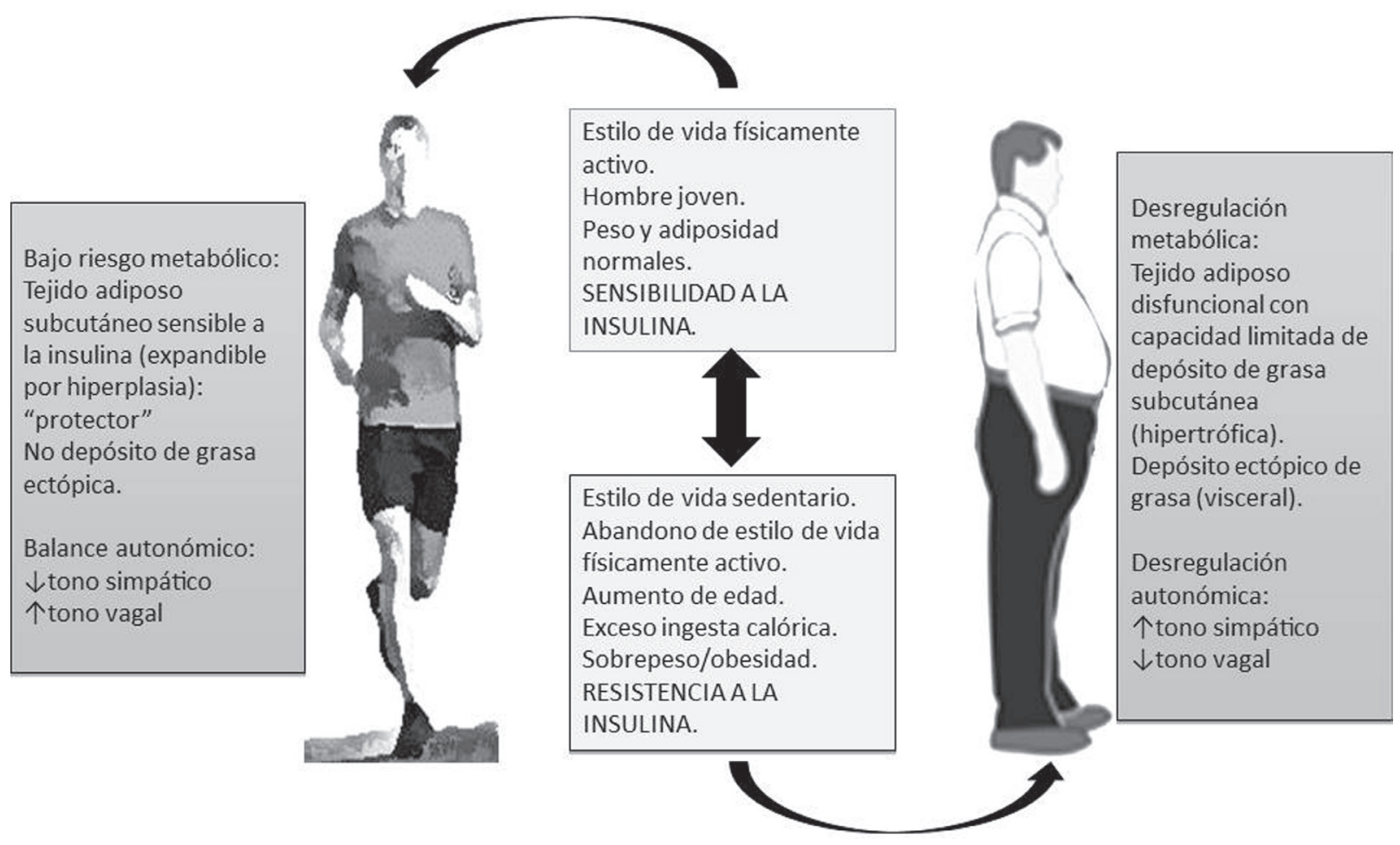

FIGURA 1. Modelo explicativo del papel de la grasa subcutánea como protector cardio-metabólico.

En un microambiente de sensibilidad a la insulina, en hombres jóvenes, físicamente activos, con peso y adiposidad normales, un exceso calórico puede acumularse preferentemente como tejido adiposo subcutáneo sensible a la insulina; éstas son condiciones de bajo riesgo metabólico y de adecuado balance autonómico ${ }^{44-47}$. Por el contrario, a medida que aumenta la edad, cuando se es sedentario o se abandona un estilo de vida físicamente activo, y se desarrolla sobrepeso/ obesidad, se empieza a instaurar un ambiente de resistencia a la insulina en el cual el tejido adiposo subcutáneo tiene capacidad limitada para almacenar el exceso calórico (tejido adiposo hipertrófico) ${ }^{30}$ y se empieza a producir un depósito de grasa ectópica a nivel visceral, la cual se relaciona directamente con el desarrollo de síndrome metabólico, independiente incluso de la grasa corporal total y la grasa subcutánea ${ }^{41-43}$, a la vez que se produce desregulación autonómica con predominio del tono simpático sobre el vagal. La flecha de doble sentido implica que muchos de estos "factores de riesgo metabólico" son modificables: aumento de actividad física, restricción calórica, control del peso y la adiposidad, e incluso modulación farmacológica de la sensibilidad a la insulina .

\section{CONCLUSIONES}

Los hallazgos aquí reportados en un grupo de hombres jóvenes (18-30 años) sin sobrepeso ni obesidad abdominal y físicamente activos $(n=24)$, mostraron que el volumen de actividad física y los indicadores de adiposidad abdominal (relación cintura/cadera y pliegue abdominal) son predictores de la variabilidad cardíaca en reposo, mostrando relación positiva con el tono vagal (HF) y negativa con el tono simpático (LF y LF/HF). Estos hallazgos son opuestos a los descritos previamente en personas con sobrepeso/obesidad y/o físicamente inactivas, y son sugestivos de que en hombres jóvenes, delgados y físicamente activos un mejor control parasimpático y la capacidad de depositar el exceso calórico en forma de grasa subcutánea pueden ser mecanismos protectores contra el desarrollo de enfermedades cardiovasculares y metabólicas. A diferencia de las personas con sobrepeso/obesidad y/o inactividad física, en las que el predominio simpático 
¿Es la grasa abdominal subcutánea un predictor de la variabilidad cardíaca en hombres con bajo riesgo metabólico?

se asocia con saturación de la capacidad de depósito de grasa a nivel subcutáneo y un mayor depósito de grasa ectópica, como factores que pueden mediar la fisiopatología de trastornos cardio-metabólicos.

\section{REFERENCIAS}

1. Indumathy J, Pal GK, Pal P, Ananthanarayanan $\mathrm{PH}$, Parija SC, Balachander J, et al. Association of sympathovagal imbalance with obesity indices, and abnormal metabolic biomarkers and cardiovascular parameters. Obes Res Clin Pract. 2015; 9(1): 55-66. DOI: 10.1016/j.orcp.2014.01.007.

2. Dekker JM, Crow RS, Folsom AR, Hannan PJ, Liao $\mathrm{D}$, Swenne CA, et al. Low heart rate variability in a 2-minute rhythm strip predicts risk of coronary heart disease and mortality from several causes: the ARIC Study. Atherosclerosis Risk In Communities. Circulation. 2000; 102(11): 1239-1244. DOI: 10.1161/01.CIR.102.11.1239.

3. Molfino A, Fiorentini A, Tubani L, Martuscelli M, Rossi Fanelli F, Laviano A. Body mass index is related to autonomic nervous system activity as measured by heart rate variability. Eur J Clin Nutr. 2009; 63(10): 1263-1265. DOI: 10.1038/ ejcn.2009.35.

4. Pal GK, Chandrasekaran A, Hariharan AP, Dutta TK, $\mathrm{Pal} \mathrm{P}$, Nanda N, et al. Body mass index contributes to sympathovagal imbalance in prehypertensives. BMC Cardiovasc Disord. 2012; 12:54. DOI: 10.1186/1471-2261-12-54.

5. Yi SH, Lee K, Shin D-G, Kim JS, Ki H-C. Differential association of adiposity measures with heart rate variability measures in Koreans. Yonsei Med J. 2013; 54(1): 55-61. DOI: 10.3349/ ymj.2013.54.1.55.

6. Millis RM, Austin RE, Hatcher MD, Bond V, Faruque MU, Goring KL, et al. Association of body fat percentage and heart rate variability measures of sympathovagal balance. Life Sci. 2010; 86: 153157. DOI: $10.1016 /$ j.lfs.2009.11.018.

7. Chintala KK, Krishna BH, N MR. Heart rate variability in overweight health care students: correlation with visceral fat. $J$ Clin Diagn Res. 2015; 9(1): CC06-CC08. DOI: 10.7860/ JCDR/2015/12145.5434.

8. Alvarez GE, Ballard TP, Beske SD, Davy KP. Subcutaneous obesity is not associated with sympathetic neural activation. Am J Physiol - Heart Circ Physiol. 2004; 287(1): 414-418. DOI: 10.1152/ ajpheart.01046.2003.

9. Blair SN. Physical inactivity: the biggest public health problem of the 21 st century. Br J Sports Med.
2009; 43(1): 1-2. DOI: 10.1136/bjsm.2008.054825.

10. Sattelmair J, Pertman J, Ding EL, Kohl HW, Haskell W, Lee I-M. Dose response between physical activity and risk of coronary heart disease: a metaanalysis. Circulation. 2011; 124(7): 789-795. DOI: 10.1161/CIRCULATIONAHA.110.010710.

11. Rennie KL, Hemingway H, Kumari M, Brunner E, Malik M, Marmot M. Effects of moderate and vigorous physical activity on heart rate variability in a British Study of civil servants. Am J Epidemiol. 2003; 158(2): 135-143. DOI: 10.1093/aje/kwg120.

12. Recomendaciones mundiales sobre la actividad física para la salud. Organización Mundial de la Salud.

13. Onis M de, Habicht JP. Anthropometric reference data for international use: recommendations from a World Health Organization Expert Committee. Am J Clin Nutr. 1996; 64(4): 650-658.

14. Wang M, Nishizawa MK, Sakamoto Y, Heymfiel SF. Japanese-American Differences in visceral adiposity and a simplified estimation method for visceral adipose tissue. In: 2004: 518 - P.

15. World Health. Waist circumference and waisthip ratio : report of a WHO expert consultation, Geneva, 8-11

16. Keklund G, Akerstedt T. Objective components of individual differences in subjective sleep quality. J Sleep Res. 1997; 6(4): 217-220. DOI: 10.1111/ j.1365-2869.1997.00217.x.

17. Malliani A, Lombardi F, Pagani M. Power spectrum analysis of heart rate variability: a tool to explore neural regulatory mechanisms. Br Heart J. 1994; 71(1): 1-2. DOI: 10.1136/hrt.71.1.1.

18. Karason K, Mølgaard H, Wikstrand J, Sjöström L. Heart rate variability in obesity and the effect of weight loss. Am J Cardiol. 1999; 83(8): 1242-1247. DOI: 10.1016/S0002-9149(99)00066-1.

19. Molfino A, Fiorentini A, Tubani L, Martuscelli M, Rossi Fanelli F, Laviano A. Body mass index is related to autonomic nervous system activity as measured by heart rate variability. Eur J Clin Nutr. 2009; 63(10): 1263-1265. DOI: 10.1038/ ejen.2009.35.

20. Urbina A, Dario Mendoza-Romero, Michel García, Johan Ortíz. Heart rate variability in young men: effect of overweight and physical inactivity. In: Shelby Walters, ed. Heart Rate Variability (HRV): Pronostic Significance, Risk Factors and Clinical Applications. Cardiology research and clinical developments. Nova Science Publishers; 2015:139-148.

21. La Rovere MT, Bigger JT, Marcus FI, Mortara A, Schwartz PJ. Baroreflex sensitivity and heart- 
rate variability in prediction of total cardiac mortality after myocardial infarction. ATRAMI (Autonomic Tone and Reflexes After Myocardial Infarction) Investigators. Lancet. 1998; 351(9101): 478-484. DOI: http://dx.doi.org/10.1016/S01406736(97)11144-8.

22. Kiviniemi AM, Hautala AJ, Seppänen T, Mäkikallio TH, Huikuri HV, Tulppo MP. Saturation of highfrequency oscillations of R-R intervals in healthy subjects and patients after acute myocardial infarction during ambulatory conditions. Am J Physiol - Heart Circ Physiol. 2004; 287(5): 19211927. DOI: 10.1152/ajpheart.00433.2004.

23. Kim JA, Park Y-G, Cho K-H, et al. Heart rate variability and obesity indices: Emphasis on the Response to Noise and Standing. J Am Board Fam Pract. 2005; 18(2): 97-103. DOI: 10.3122/ jabfm.18.2.97.

24. Melo RC, Santos MDB, Silva E, Quintero RJ, Moreno MA, Reis MS, et al. Effects of age and physical activity on the autonomic control of heart rate in healthy men. Braz J Med Biol Res. 2005; 38(9): 1331-1338. DOI:10.1590/S0100$879 \times 2005000900007$.

25. Gonçalves TR, Farinatti P de TV, Gurgel JL, da Silva Soares PP. Correlation between cardiac autonomic modulation in response to orthostatic stress and indicators of quality of life, physical capacity, and physical activity in healthy individuals. J Strength Cond Res Natl Strength Cond Assoc. 2015; 29(5): 1415-1421. DOI: 10.1519/JSC.0000000000000769.

26. Romero-Corral A, Somers VK, Sierra-Johnson J, Thomas R, Bailey k, Collazo- Clavell M, et al. Accuracy of body mass index to diagnose obesity in the US adult population. Int J Obes 2005. 2008; 32(6): 959-966. DOI: 10.1038/ijo.2008.11.

27. Pasco JA, Nicholson GC, Brennan SL, Kotowicz MA. Prevalence of obesity and the relationship between the body mass index and body fat: crosssectional, population-based Data. PLoS ONE. 2012; 7(1): e29580. DOI: 10.1371/journal.pone.0029580.

28. Windham BG, Fumagalli S, Ble A, Sollers J, Thayer JF, Najjar SS, et al. The relationship between heart rate variability and adiposity differs for Central and Overall Adiposity. J Obes. 2012; 2012: e149516. DOI: $10.1155 / 2012 / 149516$.

29. Avellaneda Pinzon SE, Urbina A. Capacidad aeróbica de bomberos aeronáuticos. Rev Univ Ind Santander Salud. 2015; 47(1): 61-67.

30. Després J-P. Body fat distribution and risk of cardiovascular disease an update. Circulation. 2012; 126(10): 1301-1313. DOI: 10.1161/ CIRCULATIONAHA.111.067264.
31. Björntorp P. Fat Cell Distribution and metabolism. Ann N Y Acad Sci. 1987; 499(1): 66-72. DOI: 10.1111/j.1749-6632.1987.tb36198.x.

32. Lear SA, James PT, Ko GT, Kumanyika S. Appropriateness of waist circumference and waistto-hip ratio cutoffs for different ethnic groups. Eur J Clin Nutr. 2010; 64(1): 42-61. DOI: 10.1038/ ejcn.2009.70.

33. Subramaniam B, Ramaswamy C. Role of gender and the Waist - Hip Ratio WHR on Heart Rate Variablility HRV in adolescent age. Natl J Physiol Pharm Pharmacol. 2013; 3(1): 4. DOI: 10.5455/ njppp.2013.3.4-8.

34. Gutin B, Howe C, Johnson MH, Humphries MC, Snieder H, Barbeau P. Heart rate variability in adolescents: relations to physical activity, fitness, and adiposity. Med Sci Sports Exerc. 2005; 37(11): 18561863. doi:10.1249/01.mss.0000175867.98628.27.

35. Schmid K, Schönlebe J, Drexler H, MueckWeymann M. Associations between being overweight, variability in heart rate, and well-being in the young men. Cardiol Young. 2010; 20(1): 5459. DOI: $10.1017 / \mathrm{S} 1047951109992009$.

36. Kim JA, Park Y-G, Cho K-H, Hong MH, Han HC, Choi YS, et al. Heart rate variability and obesity indices: emphasis on the response to noise and standing. J Am Board Fam Pract Am Board Fam Pract. 2005;18(2): 97-103.

37. Bigger JT, Fleiss JL, Steinman RC, Rolnitzky LM, Kleiger RE, Rottman JN. Frequency domain measures of heart period variability and mortality after myocardial infarction. Circulation. 1992; 85(1): 164-171. DOI: 10.1161/01.CIR.85.1.164.

38. Shi H, Song CK, Giordano A, Cinti S, Bartness TJ. Sensory or sympathetic white adipose tissue denervation differentially affects depot growth and cellularity. Am J Physiol - Regul Integr Comp Physiol. 2005; 288(4): R1028-R1037. DOI: 10.1152/ajpregu.00648.2004.

39. Kreier F, Fliers E, Voshol PJ, van Eden CG, Havekes LM, kalsbeek A, et al. Selective parasympathetic innervation of subcutaneous and intra-abdominal fat - functional implications. J Clin Invest. 2002; 110(9): 1243-1250. DOI: 10.1172/JCI15736.

40. Miyato H, Kitayama J, Hidemura A, Ishigami H, Kaisaki S, Nagawa H. Vagus nerve preservation selectively restores visceral fat volume in patients with early gastric cancer who Underwent Gastrectomy. J Surg Res. 2012; 173(1): 60-67. DOI: 10.1016/j.jss.2010.08.040.

41. Preis SR, Massaro JM, Robins SJ, Hoffmann U, Vasan RS, Irlbeck T, et al. Abdominal subcutaneous and visceral adipose tissue and insulin resistance 
¿Es la grasa abdominal subcutánea un predictor de la variabilidad cardíaca en hombres con bajo riesgo metabólico?

in the Framingham heart study. Obes Silver Spring Md. 2010; 18(11): 2191-2198. DOI:10.1038/ oby. 2010.59 .

42. Liu J, Fox CS, Hickson D, Bidulescu A, Carr JJ, Taylor HA. Fatty liver, abdominal visceral fat, and cardiometabolic risk Factors The Jackson Heart Study. Arterioscler Thromb Vasc Biol. 2011; 31(11): 2715-2722. doi:10.1161/ATVBAHA.111.234062.

43. Pou KM, Massaro JM, Hoffmann U, Vasan RS, Maurovich-Horvat P, Larson MG, et al. Visceral and subcutaneous adipose tissue volumes are crosssectionally related to markers of inflammation and oxidative stress The Framingham Heart Study. Circulation. 2007;116(11):1234-1241. DOI: 10.1161/CIRCULATIONAHA.107.710509.

44. Kim J-Y, van de Wall E, Laplante M, Azzara A, trujillo Me, Hofmann SM, et al. Obesity-associated improvements in metabolic profile through expansion of adipose tissue. J Clin Invest. 2007; 117(9): 2621-2637. DOI: 10.1172/JCI31021.

45. Porter SA, Massaro JM, Hoffmann U, Vasan RS, O’Donnel CJ, Fox CS. Abdominal subcutaneous adipose tissue: A protective fat depot? diabetes care. 2009; 32(6): 1068-1075. DOI: $10.2337 / \mathrm{dc} 08-$ 2280.

46. Bays HE, Fox KM, Grandy S, SHIELD Study Group. Anthropometric measurements and diabetes mellitus: clues to the "pathogenic" and "protective" potential of adipose tissue. Metab Syndr Relat Disord. 2010; 8(4): 307-315. DOI: 10.1089/ met.2009.0089.

47. Golan R, Shelef I, Rudich A, Gepner Y, Shemesh E, Chassidim Y, et al. Abdominal superficial subcutaneous fat : a putative distinct protective fat subdepot in type 2 diabetes. Diabetes Care. 2012; 35(3): 640-647. DOI: 10.2337/dc11-1583.

48. Kim JK, Gavrilova O, Chen Y, Reitman ML, Shulman GI. Mechanism of Insulin Resistance in A-ZIP/F-1 Fatless Mice. J Biol Chem. 2000; 275(12): 84568460. DOI: $10.1074 /$ jbc.275.12.8456.

49. Gavrilova O, Marcus-Samuels B, Graham D, Kim JK, Shulman GI, Castle AL, et al. Surgical implantation of adipose tissue reverses diabetes in lipoatrophic mice. J Clin Invest. 2000; 105(3): 271278. DOI: 10.1172/JCI7901.

50. Després J-P, Lemieux I. Abdominal obesity and metabolic syndrome. Nature. 2006; 444(7121): 881887. DOI: 10.1038/nature05488.

51. Cancello R, Zulian A, Gentilini D, Maestrini S, Della Barba A, Invitti C, et al. Molecular and morphologic characterization of superficial- and deep-subcutaneous adipose tissue subdivisions in human obesity. Obes Silver Spring Md. 2013;
21(12): 2562-2570. DOI: 10.1002/oby.20417.

52. Kim S-H, Chung J, Song S-W, Jung WS, Lee Y-A, Kim H-N. Relationship between deep subcutaneous abdominal adipose tissue and metabolic syndrome: a case control study. Diabetol Metab Syndr. 2016; 8. DOI: 10.1186/s13098-016-0127-7.

53. Alvarez GE, Ballard TP, Beske SD, Davy KP. Subcutaneous obesity is not associated with sympathetic neural activation. Am J Physiol Heart Circ Physiol. 2004; 287(1): H414-H418. DOI: 10.1152/ajpheart.01046.2003.

54. Miyazaki Y, Mahankali A, Matsuda M, Mahankali S, Hardies J, Cusi K, et al. Effect of pioglitazone on abdominal fat distribution and insulin sensitivity in type 2 diabetic patients. J Clin Endocrinol Metab. 2002; 87(6): 2784-2791. DOI: 10.1210/ jcem.87.6.8567. 